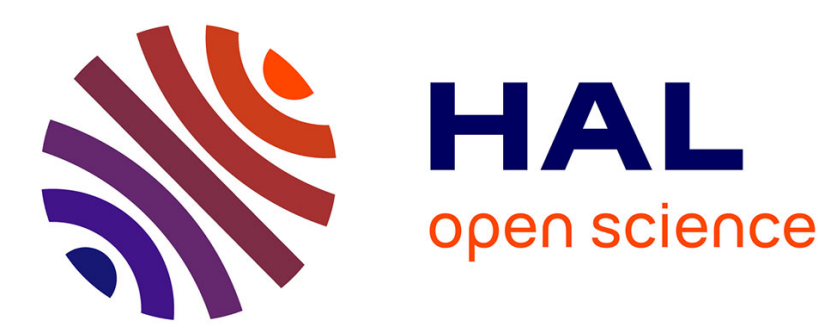

\title{
Feedback stabilization of an oscillating vertical cylinder by $P O D$ reduced-order model
}

Gilles Tissot, Laurent Cordier, Bernd Noack

\section{To cite this version:}

Gilles Tissot, Laurent Cordier, Bernd Noack. Feedback stabilization of an oscillating vertical cylinder by POD reduced-order model. CFM 2015 - 22ème Congrès Français de Mécanique, Aug 2015, Lyon,

France. hal-03446135

\author{
HAL Id: hal-03446135 \\ https://hal.science/hal-03446135
}

Submitted on 24 Nov 2021

HAL is a multi-disciplinary open access archive for the deposit and dissemination of scientific research documents, whether they are published or not. The documents may come from teaching and research institutions in France or abroad, or from public or private research centers.
L'archive ouverte pluridisciplinaire HAL, est destinée au dépôt et à la diffusion de documents scientifiques de niveau recherche, publiés ou non, émanant des établissements d'enseignement et de recherche français ou étrangers, des laboratoires publics ou privés. 


\title{
Feedback stabilization of an oscillating vertical cylinder by POD Reduced-Order Model
}

\author{
G. TISSOT, L. CORDIER and B. R. NOACK \\ Institut PPRIME, CNRS - Université de Poitiers - ENSMA \\ F86036 Poitiers cedex, France \\ Laurent.Cordier@univ-poitiers.fr
}

\section{Résumé :}

L'objectif est de démontrer que l'utilisation de modèles d'ordre réduit basés sur la Décomposition Orthogonale aux Valeurs Propres (POD) permet de stabiliser l'écoulement autour d'un cylindre circulaire en oscillation verticale et en régime laminaire (nombre de Reynolds égal à 60). Les équations de NavierStokes $2 D$ sont d'abord résolues par éléments finis en introduisant le mouvement du cylindre via la méthode ALE. Puisqu'en interaction fluide-structure, il n'est pas possible d'appliquer directement l'algorithme POD, nous avons implémenté la méthode des domaines fictifs proposée par Glowinski et al. (1999) où le domaine solide est traité comme un domaine fluide soumis à une contrainte additionnelle. Le modèle réduit POD est classiquement obtenu par projection des équations de Navier-Stokes sur les premiers modes POD. A ce niveau, le déplacement du cylindre est imposé dans le modèle réduit par introduction de multiplicateurs de Lagrange. Pour déterminer la vitesse verticale optimale du cylindre, l'algorithme Linear Quadratic Regulator est employé. Après linéarisation du modèle réduit POD autour de la solution stationnaire de l'écoulement, le gain optimal de retour d'état est obtenu comme solution d'une équation de Riccati algébrique généralisée. Finalement, quand le contrôle optimal en retour d'état est appliqué, nous montrons que l'écoulement converge rapidement vers la solution stationnaire. Par ailleurs, un contrôle évanescent est obtenu ce qui prouve l'efficacité de la technique de contrôle.

\section{Abstract :}

The objective is to demonstrate the use of reduced-order models (ROM) based on proper orthogonal decomposition (POD) to stabilize the flow over a vertically oscillating circular cylinder in the laminar regime (Reynolds number equal to 60). The 2D Navier-Stokes equations are first solved with a finite element 
method, in which the moving cylinder is introduced via an ALE method. Since in fluid-structure interaction, the POD algorithm cannot be applied directly, we implemented the fictitious domain method of Glowinski et al. (1999) where the solid domain is treated as a fluid undergoing an additional constraint. The PODROM is classically obtained by projecting the Navier-Stokes equations onto the first POD modes. At this level, the cylinder displacement is enforced in the POD-ROM through the introduction of Lagrange multipliers. For determining the optimal vertical velocity of the cylinder, a linear quadratic regulator framework is employed. After linearization of the POD-ROM around the steady flow state, the optimal linear feedback gain is obtained as solution of a generalized algebraic Riccati equation. Finally, when the optimal feedback control is applied, it is shown that the flow converges rapidly to the steady state. In addition, a vanishing control is obtained proving the efficiency of the control approach.

Mots clefs : Feedback stabilization ; Oscillating vertical cylinder ; POD Reduced-Order Model.

\section{Flow configuration}

The cylinder wake is a common generic configuration to test control methods which can be further implemented in more complex engineering applications. The stabilization of this flow consists in targeting and maintaining the unstable steady solution by control. Here, we consider the cylinder wake in fluid-structure interaction and suppose that the control is introduced by vertical oscillations of the cylinder, as sketched in figure 1 .

Let $\boldsymbol{u}$ be the velocity field and $\boldsymbol{u}^{\text {steady }}$ be the targeted steady state, the objective is to determine the best cylinder vertical velocity $V_{c}(t)$, taken as control parameter, such that we minimize

$$
\mathcal{J}\left(V_{c}(t)\right)=\int_{0}^{T}\left\|\boldsymbol{u}-\boldsymbol{u}^{\text {steady }}\right\|^{2} \mathrm{~d} t+\alpha \int_{0}^{T} y_{c}^{2}(t) \mathrm{d} t+\beta \int_{0}^{T} V_{c}^{2}(t) \mathrm{d} t,
$$

where $y_{c}$ is the cylinder vertical position. The cost functional $\mathcal{J}$ is regularized to avoid very large, then unphysical, controls. Indeed, the role of the parameter $\alpha$ is to penalize too large cylinder displacements and then to enforce the cylinder to stay near the central position, while $\beta$ penalizes too strong control actions.

\section{Linear control framework}

The determination of the control law is done with a linear control theory approach (Tissot, 2014). This framework relies on the existence of a linear model 


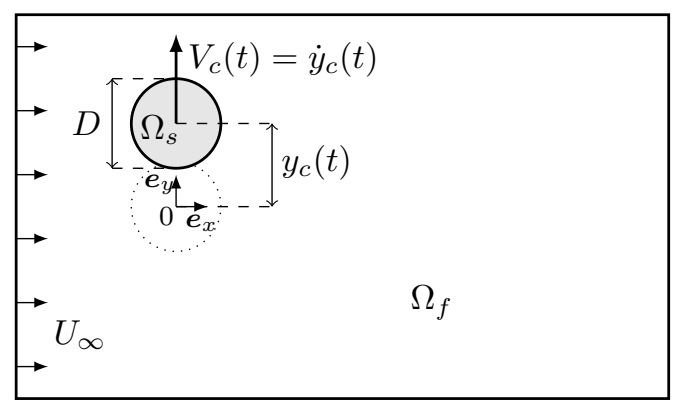

Figure 1: Cylinder wake in vertical oscillation.

$E \frac{\mathrm{d} \boldsymbol{z}}{\mathrm{d} t}=A \boldsymbol{z}+B \boldsymbol{c}$, where $\boldsymbol{z}$ is the state of the system and $\boldsymbol{c}$ the control law to be determined. Considering that the state of the system is fully and perfectly known, the linear quadratic regulator (LQR) method is considered. The objective of the LQR problem is to determine the best control law $\boldsymbol{c}$, such that the cost functional

$$
\mathcal{J}^{L Q R}(\boldsymbol{z}, \boldsymbol{c})=\frac{1}{2} \int_{0}^{T}\|F \boldsymbol{z}(t)\|^{2} \mathrm{~d} t+\frac{1}{2} \int_{0}^{T}\|\boldsymbol{c}(t)\|_{R}^{2} \mathrm{~d} t
$$

is minimized. In (2), $F$ is a matrix defining the objective to minimize, and $R$ penalizes the control $\boldsymbol{c}$ through the norm $\|\cdot\|_{R}^{2}=(R \cdot, \cdot)$ where $(\cdot, \cdot)$ denotes the inner product. The feedback control law that minimizes the value of $\mathcal{J}^{L Q R}$ is $\boldsymbol{c}=K \boldsymbol{z}$ where the feedback gain $K$ is found after solving a Riccati equation related to (2). For an infinite time horizon, the minimization of (2) leads to the generalized algebraic Riccati equation (GARE) given by

$$
A^{*} X E+E^{*} X A-E^{*} X B R^{-1} B^{*} X E+F F^{*}=0,
$$

where $A^{*}$ is the adjoint matrix of $A$. The optimal feedback gain is then $K=$ $-R^{-1} B^{*} X E$ where $X$ is solution of (3). For large-scale problems, as the one arising from spatial discretization of the Navier-Stokes equations with finite element methods, the resolution of the GARE given by (3) is still unfeasible (Benner and Saak, 2010). One way is to obtain low-rank solutions of GAREs based on simulations of linear systems coupled with Proper Orthogonal Decomposition (POD) (see Kramer and Singler, 2014, for instance). An alternative is to derive a reduced-order model (ROM) for the dynamics (Ravindran, 2007). 


\section{Flow modelling for optimal control in fluid- structure interaction}

\subsection{Fictitious domain approach}

For the fluid-structure interaction problem, we consider a Lagrange-multiplierbased fictitious-domains method as in Glowinski et al. (1999). For that, let the whole computational domain $\Omega=\Omega_{f}(t) \cup \Omega_{s}(t)$ be constituted by the fluid domain $\Omega_{f}(t)$ and by an immersed body $\Omega_{s}(t)$, with $\Omega_{f}(t) \cap \Omega_{s}(t)=\emptyset$. The principle is to consider the whole domain as a fluid, governed by the Navier-Stokes equations, and to treat the solid in $\Omega_{s}(t)$ as a fluid subject to an additional constraint. The full system is then defined as

$$
\left\{\begin{array}{l}
\frac{\partial \boldsymbol{u}}{\partial t}+(\boldsymbol{u} . \nabla) \boldsymbol{u}=-\nabla p+\frac{1}{R e} \Delta \boldsymbol{u} \quad \forall \boldsymbol{x} \in \Omega \\
\nabla \cdot \boldsymbol{u}=0 \quad \forall \boldsymbol{x} \in \Omega \\
\boldsymbol{u}(\boldsymbol{x}, t)=\left(\begin{array}{c}
0 \\
V_{c}(t)
\end{array}\right) \quad \forall \boldsymbol{x} \in \Omega_{s}(t) .
\end{array}\right.
$$

The cylinder velocity constraint in $\Omega_{s}(t)$ has still to be enforced. The system (4) can be numerically solved by enforcing the constraint with Lagrange multipliers (Glowinski et al., 1999). The linearization of (4) around the steady state $\boldsymbol{u}^{\text {steady }}$ leads to a high-fidelity model that is too large for the direct resolution of (3). For this reason, a step of model reduction is required before searching for an optimal control law.

\subsection{Model reduction in fluid-structure interaction}

For enforcing the boundary conditions, the strategy is to first derive a reducedorder model by Galerkin projection of (4) onto the POD modes (Tissot, 2014), and then to apply to this model the constraint of the domain motion expressed in the POD subspace (Kalashnikova and Barone, 2012). The weak form of (4) obtained by Galerkin projection onto the POD modes provides a drastic reduction of the number of degrees of freedom. The Navier-Stokes equations being satisfied on the full domain $\Omega$, (4) is projected onto the $N_{\text {gal }}$ first POD modes which are properly defined on $\Omega$ (Liberge and Hamdouni, 2010). For the cylinder motion constraint, $N_{c}=3$ arbitrary test functions vanishing on $\Omega_{f}(t)$ are used. These functions are chosen to enforce the streamwise and transversal mean velocities and the mean rotation in $\Omega_{s}(t)$. Finally, it leads to a $N_{c^{-}}$ dimensional time-dependent linear system of constraints which is enforced by the introduction of a Lagrange multiplier $\boldsymbol{\lambda}(t)=\left(\lambda_{1}, \ldots, \lambda_{N_{c}}\right)^{T}$. The reduced system can be written as a low-order constant-linear-quadratic descriptor system 
given by:

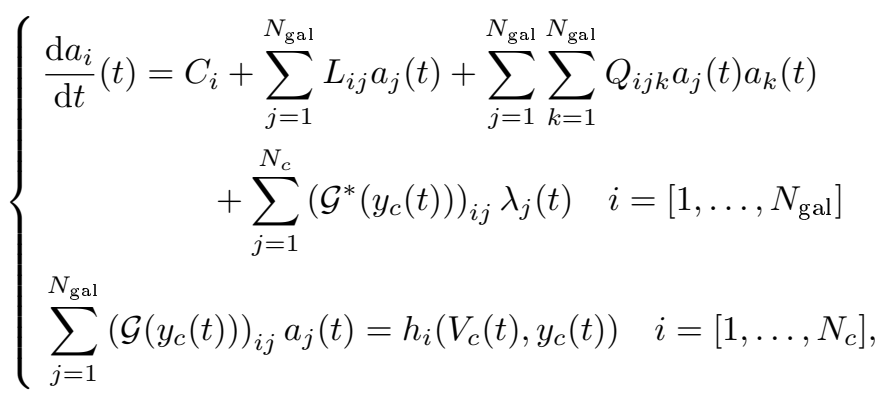

where $a_{i}\left(i=1, \cdots, N_{\text {gal }}\right)$ are the time coefficients of the POD modes.

\section{Results}

\subsection{POD Reduced-Order Model}

Snapshots of the cylinder undergoing vertical oscillations have been generated using a moving mesh implemented in COMSOL Multiphysics 4.3 through an ALE formulation. The forced numerical simulation is initialized with the steady state solution $\boldsymbol{u}^{\text {steady }}$. For the cylinder, the velocity $V_{c}$ and the position $y_{c}$ are chosen such that the system first undergoes from $t=0$ to $t=100$ a transient regime from the steady state to an established unactuated state. Finally, the flow is actuated from $t=100$ to $t=200$ at the natural frequency. After interpolation on a regular mesh and subtraction of the steady state, the POD analysis is performed on the data (Tissot, 2014). A Galerkin projection of the Navier-Stokes equations onto the first 47 POD modes is performed. According to section 3.2, we then add the constraints of mean vertical, horizontal and tangential velocities inside the solid domain. Figure 2 represents the time coefficients obtained as solutions of (5) by comparison with the temporal POD eigenfunctions. We observe that the ROM's dynamics in the transient, established and actuated regimes are very close to the original dynamics. In conclusion, the constrained ROM (5) is considered to represent with a sufficient accuracy the oscillating vertical cylinder.

\subsection{Feedback control}

After linearization of (5) around the steady state, the optimal feedback gain expressed in the POD subspace is determined as solution of the low-order GARE (3). By construction, this feedback gain is designed to stabilize the ROM (5), and not the high-fidelity model (4). However, this feedback gain should be a good approximation of the optimal linear feedback gain solution of the highorder GARE (3) related to (4). To check this hypothesis, we expressed this gain 

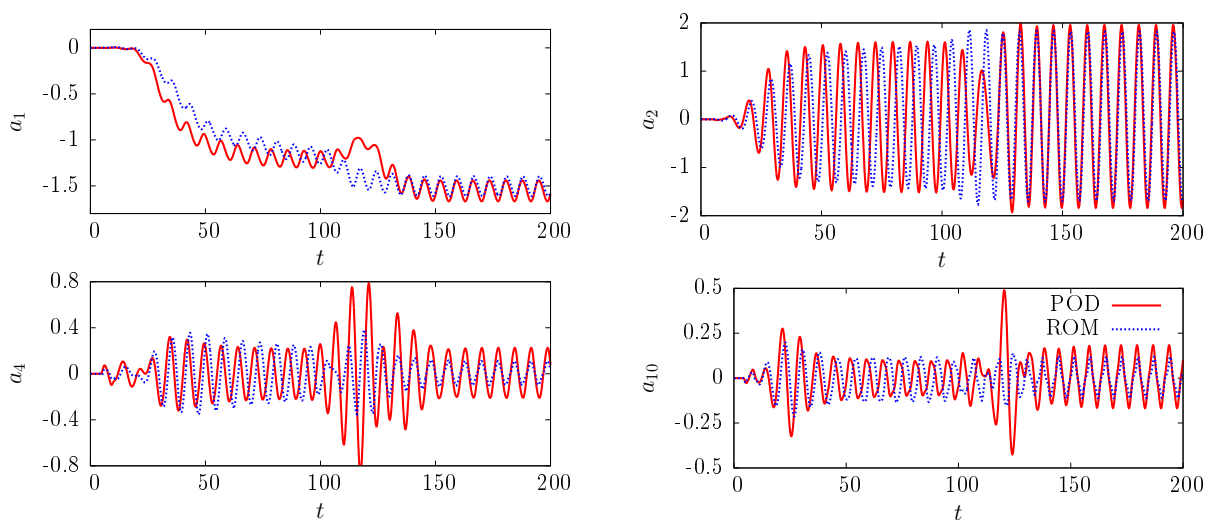

Figure 2: POD ROM of the vertical oscillating cylinder at $R e=60$. The time coefficients obtained by projection on the POD modes are in red, whereas the solutions of the POD ROM (5) are represented in blue.

in the high-order space and used the result to control the high-fidelity model (4). This control design leads to a stabilization of the cylinder wake at $R e=60$ as shown in figure 3 .

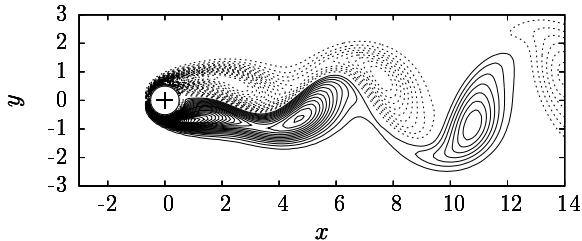

(a) Natural flow.

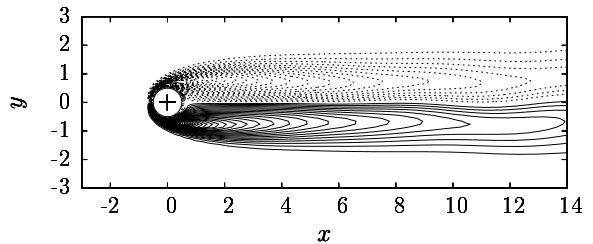

(b) Flow controlled by cylinder vertical oscillation.

Figure 3: Linear feedback stabilization of the wake behind a circular cylinder at $R e=60$. Contour lines of vorticity. The dashed lines correspond to negative values.

\section{Conclusion}

An efficient feedback control has been developed in a moving domain configuration. The domain motion information has been incorporated in the POD ROM enforcing an additional constraint by Lagrange multipliers. This strategy allows the use of physical model-based control for large-scale problems in fluid-structure interaction. We have successfully tested this control design on the stabilization of a circular cylinder wake by vertical oscillations at $R e=60$. 


\section{References}

P. Benner and J. Saak. A Galerkin-Newton-ADI method for solving large-scale algebraic Riccati equations. Preprint SPP1253-090, DFGSPP1253, 2010.

R. Glowinski, T. W. Pan, T. I. Hesla, and D. D. Joseph. A distributed Lagrange multiplier/fictitious domain method for particulate flows. International Journal of Multiphase Flow, 25(5):755-794, 1999.

I. Kalashnikova and M. F. Barone. Efficient non-linear proper orthogonal decomposition/Galerkin reduced order models with stable penalty enforcement of boundary conditions. International Journal for Numerical Methods in Engineering, 90(11):1337-1362, 2012.

B. Kramer and J. R. Singler. A POD Projection Method for Large-Scale Algebraic Riccati Equations. Submitted for publication, 2014.

E. Liberge and A. Hamdouni. Reduced order modelling method via proper orthogonal decomposition (POD) for flow around an oscillating cylinder. Journal of Fluids and Structures, 26(2):292-311, 2010.

S. S. Ravindran. Optimal boundary feedback flow stabilization by model reduction. Computer Methods in Applied Mechanics and Engineering, 196(25-28): 2555-2569, 2007.

G. Tissot. Réduction de modèle et contrôle d'écoulements. PhD thesis, Université de Poitiers, 2014. 